Observatorio 



\title{
¿Hacia una contrarreforma de pensiones? Notas para el Pacto de Toledo*
}

\author{
ÁNGEL DE LA FUENTE*** \\ FEDEA e Instituto Económico, CSIC \\ MIGUEL ÁNGEL GARCÍA DÍAZ*** \\ Universidad Rey Juan Carlos y FEDEA \\ ALFONSO R. SÁNCHEZ**** \\ CUNEF y FEDEA
}

Recibido: Noviembre, 2018

Aceptado: Abril, 2019

\section{Resumen}

En este artículo se analizan las posibles consecuencias de la derogación de la reforma de 2013 de la normativa sobre pensiones. Con este fin, se construyen proyecciones de los ingresos y gastos del sistema de pensiones contributivas de la Seguridad Social hasta 2070 con y sin dicha reforma y se exploran sus implicaciones para las cuentas públicas y para el reparto de la renta entre activos y pensionistas bajo dos escenarios alternativos sobre el influjo de inmigrantes en las próximas décadas.

Palabras clave: reforma de pensiones, sostenibilidad.

Clasificación JEL: H55, J11.

\section{Introducción}

La reforma de 2013 de la Seguridad Social introdujo dos mecanismos de ajuste automático que buscaban garantizar la sostenibilidad financiera del sistema público de pensiones español. El primero de ellos, conocido como factor de sostenibilidad, ligaba la cuantía inicial de las pensiones a la evolución de la esperanza media de vida en el momento de la jubilación. El segundo, el lla-

\footnotetext{
* Este trabajo ha sido financiado en parte por el Ministerio de Economía, Industria y Competitividad a través del proyecto ECO2017-87862-P. Agradecemos los comentarios y sugerencias de Rafael Doménech y Mariano Guindal.

** ORCID ID: 0000-0002-5738-5162.

*** ORCID ID: 0000-0003-3290-2415.

**** ORCID ID: 0000-0002-3815-6540.
} 
mado Índice de Revalorización de las Pensiones (IRP), intentaba evitar que los gastos del sistema excediesen permanentemente a sus ingresos por el procedimiento de ligar la tasa de actualización anual de las pensiones ya en vigor con la situación financiera (observada y prevista) del sistema a medio plazo, abandonando la referencia tradicional del Índice de Precios al Consumo (IPC).

El acuerdo alcanzado por el anterior Gobierno del PP con el PNV para la aprobación de los Presupuestos Generales del Estado de 2018 dejó a la reforma de 2013 en una situación muy precaria. En principio, el acuerdo establecía un paréntesis en la aplicación de la reforma, aplazando la entrada en vigor del factor de sostenibilidad hasta un máximo de cinco años y suspendiendo temporalmente la aplicación del IRP para revalorizar las pensiones durante 2018 y 2019 a una tasa del 1,6\% anual, próxima la inflación esperada y muy superior al $0,25 \%$ que establecía la norma suspendida en las circunstancias actuales. En la práctica, además, todo apunta a que el cambio de rumbo será permanente pues prácticamente ningún partido defiende ahora la necesidad de contener el crecimiento del gasto en pensiones y parece existir un cierto consenso entre ellos, aunque con matices, sobre la necesidad de recuperar el IPC, al menos como referencia principal, para la revisión anual de las pensiones.

En este artículo se argumenta que desmantelar la reforma de 2013 no es buena idea, y mucho menos aún si se hace sin contar con un plan realista y detallado de cómo se piensa garantizar la equidad intergeneracional y la sostenibilidad financiera del sistema de pensiones durante las próximas tres décadas, en las que nos enfrentaremos a circunstancias demográficas muy adversas para las finanzas del sistema como resultado de la llegada a la jubilación de la generación del baby boom, la caída de la natalidad y el incremento de la esperanza de vida. El resto del trabajo se divide en tres secciones. La sección 2 se dedica al complicado reto demográfico que afrontará nuestro país durante las próximas décadas, destacando la elevada incertidumbre sobre la evolución de la población que resulta principalmente de la dificultad de predecir los movimientos migratorios. En la sección 3 se presentan proyecciones de los ingresos y gastos del sistema de pensiones contributivas de la Seguridad Social con y sin la reforma de 2013 y se exploran sus implicaciones para nuestras cuentas públicas bajo dos escenarios migratorios alternativos. Las proyecciones se construyen utilizando un modelo de equilibrio general dinámico desarrollado y calibrado por Sánchez (2017 a y b) para el informe sobre el sistema de pensiones que FEDEA publicó el año pasado (véase De la Fuente et al., DGS, 2017, publicado parcialmente como DGS 2018 a y b). Finalmente, la sección 4 cierra con una serie de conclusiones y recomendaciones entre las que destaca la necesidad de que la derogación de la reforma de 2013, si se confirma, vaya acompañada de medidas de contención del gasto en pensiones que habrían de extenderse también a los ya jubilados, evitando poner todo el peso de garantizar la viabilidad del sistema sobre medidas de ingreso que exigirían entonces un incremento muy notable de la presión fiscal sobre determinadas cohortes de trabajadores y podrían dejarnos sin el margen presupuestario necesario para afrontar otras necesidades sociales.

\section{El reto demográfico}

Uno de los grandes retos a los que se tendrá que enfrentar nuestro sistema público de pensiones en las próximas décadas es el rápido proceso de envejecimiento de la población es- 
pañola que comenzó a mediados de los años setenta. Las causas inmediatas de este fenómeno se ilustran en el Gráfico 1. Se trata del aumento de la esperanza de vida registrado durante las últimas décadas y, muy especialmente, del desplome de la tasa de natalidad española, que se redujo en un $60 \%$ entre mediados de los setenta y mediados de los noventa hasta situarnos entre los países con menores índices de fecundidad del mundo.

\section{Gráfico 1 \\ FACTORES SUBYACENTES AL ENVEJECIMIENTO DE LA POBLACIÓN ESPAÑOLA}

a. Tasa de fertilidad bruta de la pob. 20-40 (pob. 0/pob 20-40 un año antes)

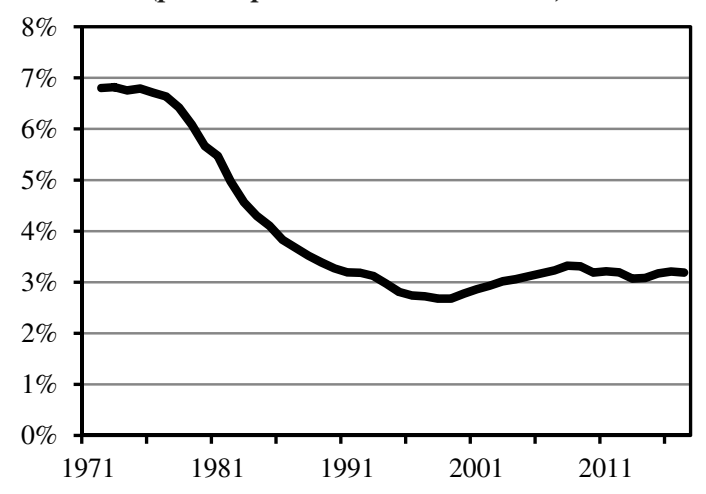

b. Esperanza de vida ${ }^{(*)}$

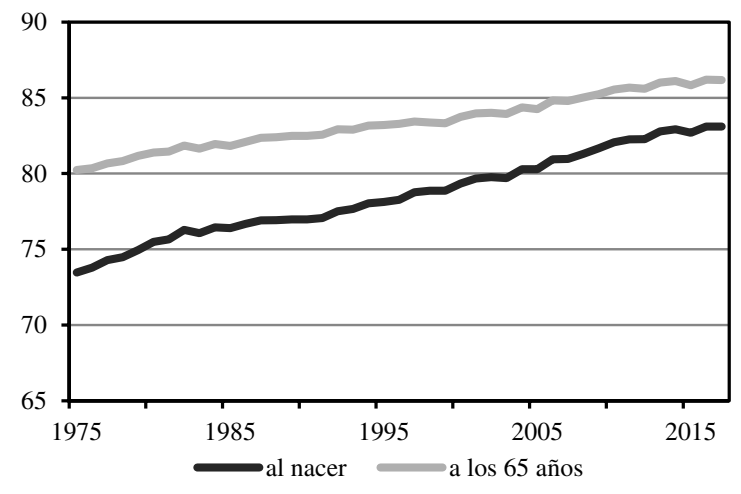

Fuente: INE (2018a y b)

(*) Lo que se dibuja en el gráfico es la edad media prevista de fallecimiento, no el tiempo esperado de vida adicional, que es lo que se suele entender por esperanza de vida.

Estos cambios en los parámetros demográficos básicos han tenido un efecto muy grande sobre la estructura de la población española y seguirán teniéndolo en las próximas décadas. El Gráfico 2 muestra la distribución de la población residente en España en 2018 de acuerdo con su fecha de nacimiento. En la curva de población resultan claramente visibles 
los efectos del desplome de la tasa de natalidad, que se traduce en un atípico patrón de cohortes de tamaño cada vez menor entre 1975 y 1995. Las líneas verticales que aparecen en el gráfico dividen a la población de acuerdo con grandes tramos de edad con límites en 18 y 65 años. Si desplazamos hacia la derecha la "ventana" que aísla a la población en edad de trabajar, podemos hacernos una idea aproximada de hacia dónde vamos. Buena parte de las cohortes más numerosas (las del llamado baby boom) ya han salido de la edad fértil y están aproximándose a la jubilación, mientras que las cohortes que entran a partir de ahora en edad de trabajar son ya y seguirán siendo durante los próximos veinticinco o treinta años de un tamaño inferior al de las que salen, poniendo presión al alza sobre el gasto en pensiones y cargando su peso sobre una base cada vez más reducida de personas en edad de trabajar.

\section{Gráfico 2 \\ POBLACIÓN RESIDENTE EN ESPAÑA EN 2018. DISTRIBUCIÓN POR FECHA DE NACIMIENTO}

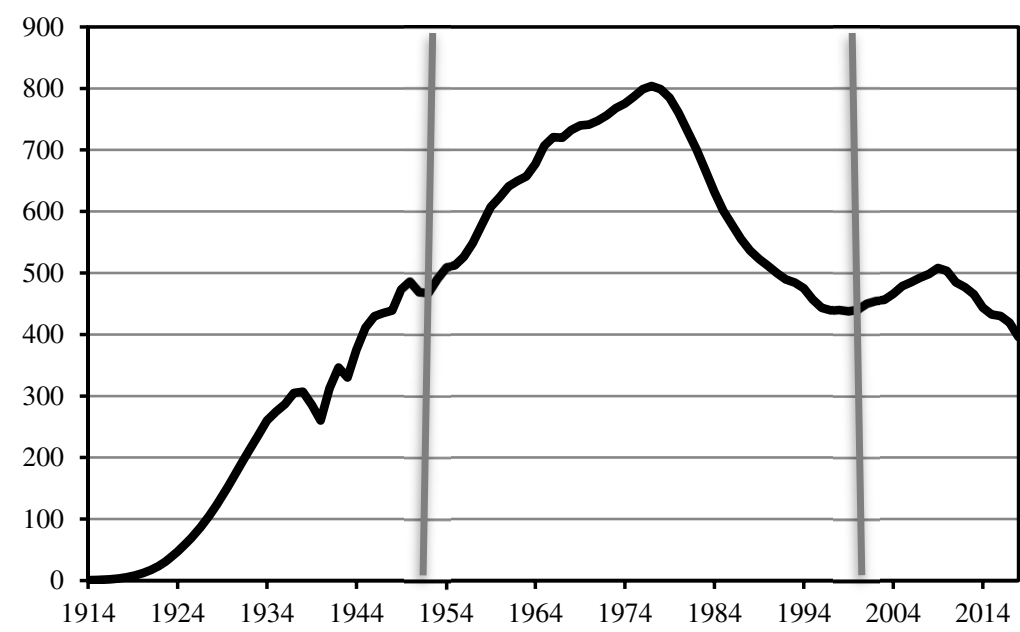

La evolución de la población y de la fuerza laboral españolas a medio plazo está predeterminada en buena parte por la estructura de la población actual que recoge el Gráfico 2, pero depende también en alguna medida del comportamiento de nuestras tasas de natalidad y mortalidad y de los flujos migratorios. Mientras que la natalidad y mortalidad suelen moverse de una forma suave y más predecible, el comportamiento de la inmigración es potencialmente mucho más volátil, lo que la convierte en el principal factor de incertidumbre demográfica durante las próximas décadas.

El Gráfico 3 resume la evolución del saldo migratorio neto de nuestro país desde 1970. El saldo es prácticamente nulo hasta 1990, aumenta durante los noventa hasta unos 150.000 efectivos al año y se dispara después durante unos años a comienzos de siglo en los que se acumulan unos 5 millones de inmigrantes, para desplomarse finalmente con la llegada de la crisis. 


\section{Gráfico 3}

FLUJOS MIGRATORIOS NETOS, ESPAÑA DESDE 1970

a. Entradas netas anuales, miles

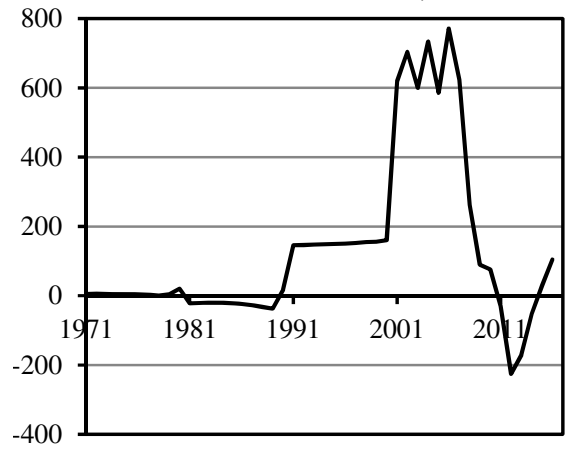

b. Totales acumulados, millones

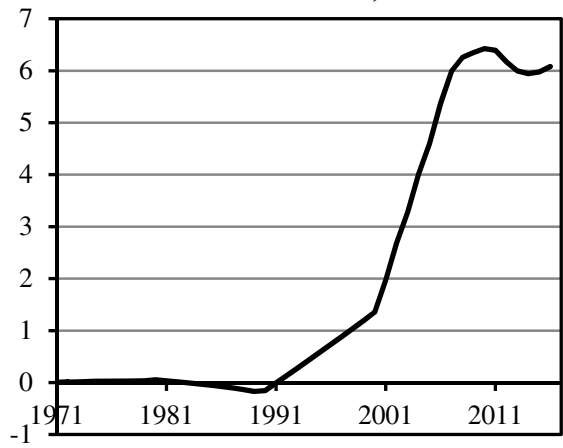

Dada la experiencia de estas últimas décadas, intentar proyectar la evolución de la inmigración resulta ciertamente arriesgado, lo que aconseja considerar un abanico de posibilidades. Las previsiones de gastos e ingresos del sistema de pensiones que comentaremos más adelante contemplan dos escenarios demográficos, uno a nuestro entender bastante realista y otro mucho más optimista en cuanto a la evolución de los flujos migratorios. El primero de ellos se basa en las proyecciones demográficas de EUROSTAT (EUROPOP2013, European Commission, 2014). En materia de inmigración, este escenario supone que el valor negativo del saldo migratorio neto español en los últimos años de la crisis se reduce gradualmente e invierte su signo a partir de 2025, con una senda ascendiente que alcanza un pico de unas 300.000 entradas netas en torno a 2050 y desciende suavemente durante el resto del período. Como alternativa, también consideramos un escenario de alta inmigración (AI) en el que se supone que la recuperación de los flujos migratorios es mucho más rápida que en el escenario base, de forma que el influjo neto de población se sitúa en torno a los 300.000 efectivos anuales a partir de 2022.

\section{Gráfico 4}

FLUJOS MIGRATORIOS NETOS EN ALGUNOS ESCENARIOS DEMOGRÁFICOS ALTERNATIVOS

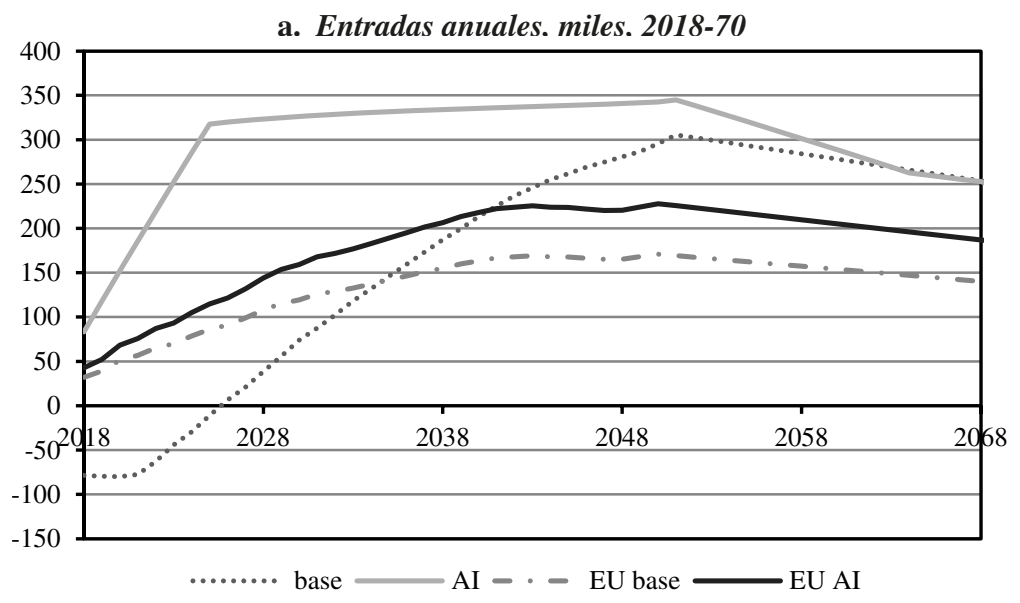


(Continuación.)

b. Totales acumulados, millones

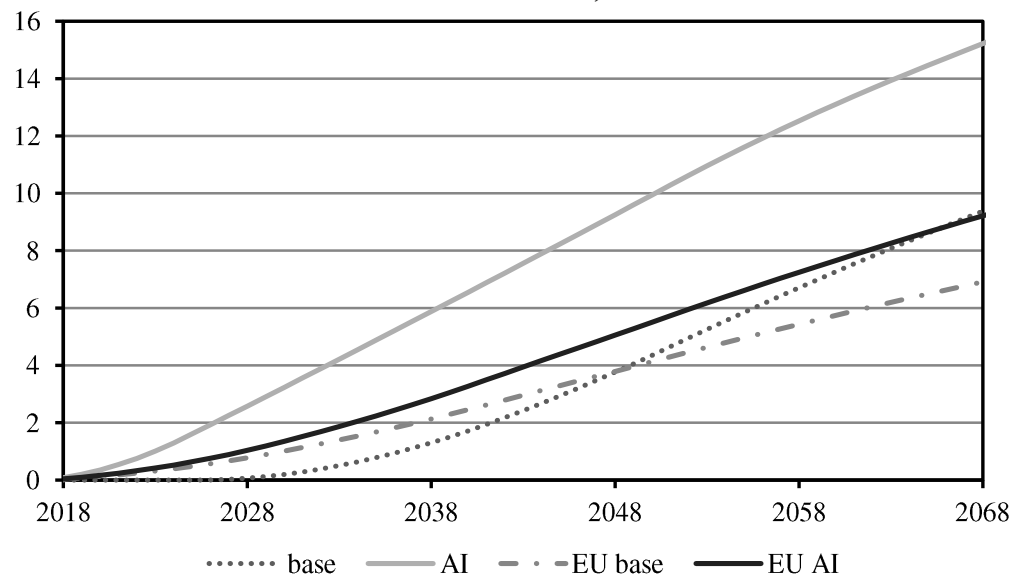

c. Entradas anuales, miles, 2018-50

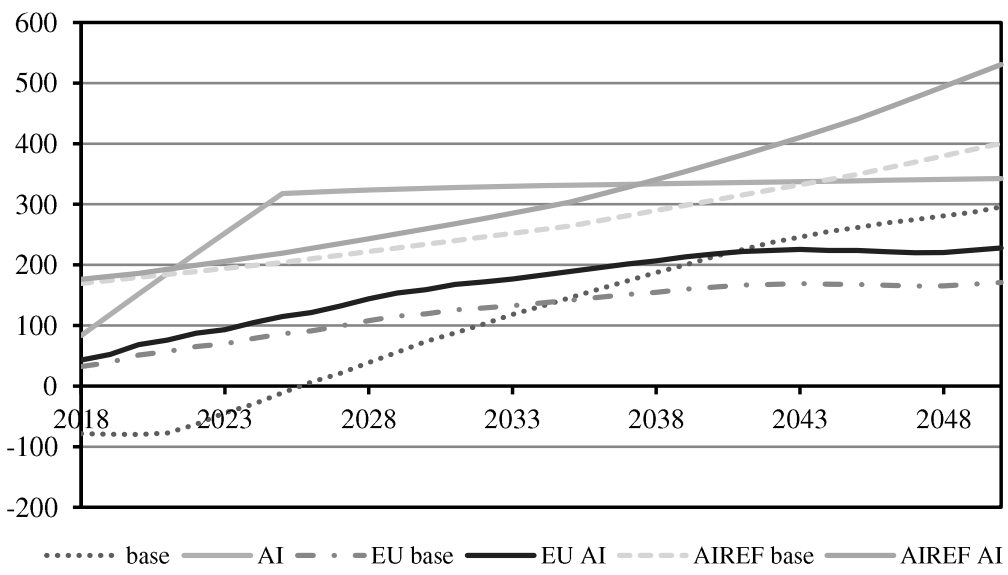

El Gráfico 4 compara ambos escenarios. Entre 2018 y 2060, la diferencia acumulada entre ellos en términos del influjo neto de inmigrantes está en torno a los 6,5 millones de personas, lo que marca la diferencia entre un apreciable aumento de la población en un caso y su práctico estancamiento en el otro. Como referencia, el Gráfico 4 muestra también las últimas previsiones migratorias de Eurostat para España, que son las que se utilizan en el último Aging Report de la Comisión Europea ${ }^{1}$, y las recientes proyecciones de la AIREF (2018) que se recogen en el panel $c$ para un período algo más corto que las demás series. Como se aprecia en el Gráfico, tanto el nuevo escenario central de Eurostat como su escenario de alta inmigración se sitúan en la zona intermedia entre nuestras dos proyecciones de entradas netas durante la primera parte del período analizado y caen después por debajo de nuestros dos 
escenarios, mientras que las proyecciones de la AIREF se sitúan cerca de nuestro escenario de inmigración elevada. Aunque el perfil temporal es diferente, la entrada neta de inmigrantes acumulada en 2050 difiere en sólo un 5\% entre los dos escenarios de alta inmigración (9,9 millones en nuestro caso vs. 10,4 en el de la AIReF). En conjunto, por tanto, nuestro escenario de alta inmigración podría acotar, al menos aproximadamente, los riesgos al alza ligados al comportamiento de los influjos inmigratorios durante las próximas décadas.

La inmigración juega un papel muy importante en cualquier proyección de las cuentas del sistema de pensiones porque tiene un efecto directo sobre la evolución de la tasa de dependencia de mayores, que es uno de los parámetros clave a estos efectos. El Gráfico 5 muestra la evolución de esta variable en nuestros dos escenarios migratorios, calculando la tasa de dependencia tanto sobre la población en edad de trabajar como sobre la población ocupada. En el escenario base, la tasa de dependencia de mayores, definida con un criterio puramente demográfico como el número de personas en edad de jubilación (65+) por persona en edad de trabajar (20-64) aumenta desde 0,32 en 2018 hasta un máximo de 0,68 en 2050, con un incremento del $113 \%$. En el escenario de alta inmigración, el incremento hasta el pico sería de "sólo" el $73 \%$, cuarenta puntos menos pero todavía muy significativo, y coincidiría casi exactamente con la previsión de la AIReF para 2050 en su escenario más optimista (en el percentil 20 de la distribución estimada de la tasa de dependencia) cuando la tasa de dependencia se calcula con la población 20-64 como aquí.

\section{Gráfico 5}

EVOLUCIÓN DE LA TASA DE DEPENDENCIA BAJO DISTINTOS ESCENARIOS MIGRATORIOS (población 65+ partida por población en edad de trabajar o población ocupada), índices con $2018=100$

a. Escenario demográfico base

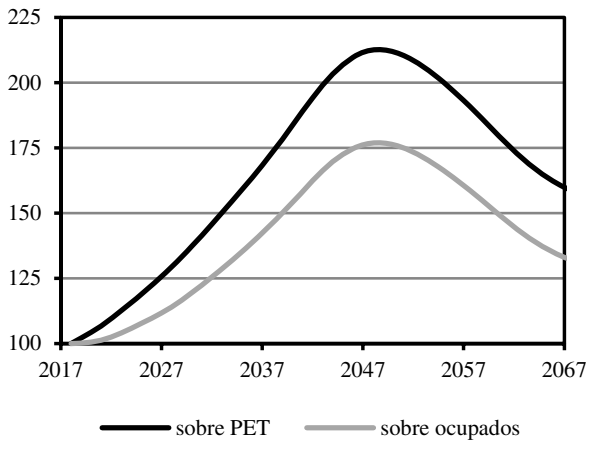

b. Escenario de alta inmigración

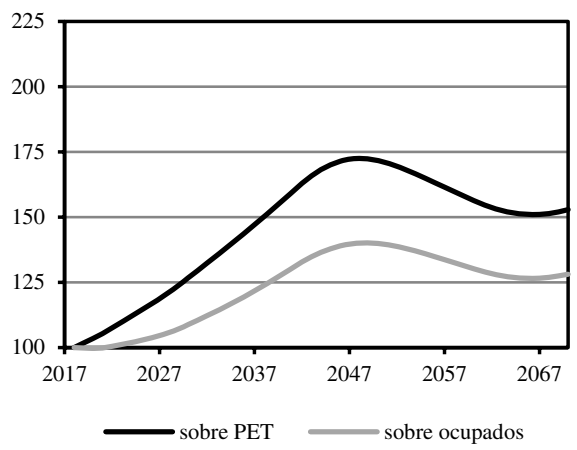

Así pues, todo apunta a que en las próximas décadas la sociedad española tendrá que enfrentarse a un importante shock demográfico que pondrá una gran presión sobre nuestro sistema de pensiones y sobre otras partidas importantes de gasto público, como la sanidad y la dependencia. Uno de los factores que podría mitigar este shock sería un incremento de las tasas de actividad y empleo de la población en edad de trabajar, lo que elevaría el número de 
trabajadores ocupados que han de hacerse cargo de cada pensión para cualquier valor dado de la tasa de dependencia demográfica. El Gráfico 5 muestra también la evolución esperada de la tasa de dependencia calculada sobre la población ocupada bajo hipótesis muy optimistas sobre la evolución de nuestro mercado de trabajo. ${ }^{2}$ Bajo estas hipótesis, el incremento de la tasa de dependencia es sensiblemente menor cuando se calcula en términos de la población ocupada en vez de la población en edad de trabajar (PET), pero el shock sigue siendo importante: estaríamos hablando, como mínimo, de un incremento de entre 40 y 77 puntos porcentuales en esta variable de aquí al 2050. En el escenario más pesimista, cada ocupado tendría que hacerse cargo del pago de 0,87 pensiones y en el más optimista de 0,68 , frente a las 0,49 que le corresponden ahora.

Un último comentario antes de abandonar el tema de la inmigración. Todos nuestros cálculos suponen implícitamente que los inmigrantes que puedan llegar en las próximas décadas serán idénticos a los nativos en términos de su nivel de formación y, más generalmente, de su nivel de productividad. Estamos suponiendo, por tanto, una inmigración con un nivel medio de cualificación relativamente elevado y un buen dominio del idioma, lo que podría no ser fácilmente alcanzable, especialmente en los escenarios con mayor inmigración. Ha de tenerse en cuenta también que el modelo que hemos utilizado no recoge ninguno de los posibles efectos disruptivos que podrían ir ligados a fuertes influjos de población procedentes de países con culturas e idiomas muy diferentes de los nuestros. Ambas omisiones han de tenerse muy en cuenta para evitar caer en la tentación de pensar que una política migratoria laxa podría ofrecer soluciones indoloras a los problemas de nuestro sistema de pensiones.

\section{Los efectos de derogar la reforma de 2013: algunas simulaciones ilustrativas}

¿Cómo afectaría a las cuentas de la Seguridad Social el desmantelamiento de la reforma de 2013? Para responder a esta cuestión, partiremos de los resultados de una serie de trabajos recientes en los que la evolución de nuestro sistema público de pensiones se simula bajo distintos escenarios económicos y demográficos. Para ello se utiliza un modelo de equilibrio general dinámico de generaciones solapadas con agentes heterogéneos en términos de su nivel educativo, género y preferencias por el ocio. El modelo incorpora una descripción muy detallada del sistema de pensiones contributivas de jubilación y viudedad junto a una modelización más estilizada de otros programas de trasferencias y gasto público que se financian con un impuesto proporcional sobre todas las rentas cuyo tipo se ajusta endógenamente para mantener sendas prefijadas de otro gasto público y de deuda en relación al PIB. El modelo se calibra cuidadosamente para reproducir la evolución de la economía española en el intervalo 2001-2014, tanto en términos de los principales agregados de Contabilidad Nacional como de ciertas pautas y parámetros demográficos y microeconómicos (perfil de ingresos a lo largo del ciclo vital, participación laboral, ahorro y riqueza acumulada). ${ }^{3}$ En al Anexo 2 se incluye un cuadro resumiendo los principales supuestos utilizados para construir el escenario base de nuestras simulaciones.

Hay que observar que, aunque la edad de jubilación se determina dentro del modelo, las tasas de actividad y ocupación de los adultos se imponen de forma exógena de acuerdo 
con las hipótesis resumidas en la nota al pie número 2. El modelo, por tanto, no recoge los posibles efectos sobre la participación y el empleo de los incrementos en impuestos que son necesarios en el modelo para mantener la trayectoria prefijada de deuda pública tras la derogación de la reforma, aunque sí captura su impacto sobre las decisiones de consumo/ahorro (y con ello sobre la evolución del stock de capital agregado, los tipos de interés y los salarios). La falta de respuesta endógena de la oferta de trabajo empuja en la dirección de hacer que nuestras previsiones sean demasiado optimistas y deban verse, por tanto, como estimaciones de mínimos. En la misma línea, el modelo se simula bajo una serie de supuestos sobre la evolución de la inflación, la productividad y otras variables macroeconómicas que tienden a ser, bastante optimistas, aunque seguramente no tanto como los que hemos hecho en materia de empleo. ${ }^{4}$ De esta forma, buscamos asegurarnos de que nuestras estimaciones de la evolución del gasto en pensiones y del coste de la derogación de la reforma de 2013 son conservadoras. Una descripción detallada de los distintos escenarios y un análisis de la sensibilidad de los resultados a posibles cambios en los supuestos más importantes puede encontrarse en DGS (2018a). ${ }^{5}$

\section{a. El escenario base: evolución del sistema con inmigración moderada y manteniendo la reforma del 2013}

Haremos en primer lugar los cálculos bajo el escenario inmigratorio que consideramos más realista, suponiendo que se mantiene la reforma de 2013 y que los tipos de cotización permanecen constantes a su nivel actual. Bajo este escenario, los mecanismos de ajuste automático introducidos en la reforma contendrían el crecimiento del gasto en pensiones durante las próximas décadas, manteniéndolo en niveles que podrían ser soportables financieramente, especialmente si se actúa pronto por el lado del ingreso, aunque también plantearían problemas de suficiencia que como mínimo exigirían medidas paliativas para las pensiones de menor cuantía. ${ }^{6}$

El Gráfico 6 resume el comportamiento de las principales variables de interés bajo este escenario. El panel $a$ muestra la evolución prevista de los ingresos y gastos del subsistema de pensiones contributivas de la Seguridad Social, medidos ambos como porcentaje del PIB. Los ingresos atribuidos al subsistema incluyen las cotizaciones sociales de ocupados y parados y las aportaciones del Estado para financiar los complementos a mínimos, netas del coste de las prestaciones contributivas distintas de las pensiones (incapacidad temporal y maternidad, fundamentalmente), así como otros ingresos menores (patrimoniales y por tasas). El gasto en pensiones contributivas incluye la parte correspondiente de los costes de gestión de la Seguridad Social además de las prestaciones pagadas. La diferencia entre gastos e ingresos así medidos rondaba en 2017 los 19.000 millones de euros o el 1,6\% del PIB. De acuerdo con nuestras proyecciones, en el escenario base los ingresos netos del sistema se mantendrían estables ligeramente por encima de1 9\% del PIB durante todo el período mientras que los gastos permanecerían prácticamente constantes hasta 2030 y aumentarían después en 1,5 puntos hasta alcanzar un pico del 12,2\% del PIB en 2050. A partir de ahí, los gastos se reducirían rápidamente con la normalización de la pirámide de población hasta llevar al sistema al equilibrio financiero en la segunda mitad de los 2060. 


\section{Gráfico 6 \\ ESCENARIO DEMOGRÁFICO BASE + MANTENIMIENTO DE LA REFORMA DE 2013 SUBSISTEMA DE PENSIONES DE LA SEGURIDAD SOCIAL CONTRIBUTIVA}

a. Gastos e ingresos/PIB

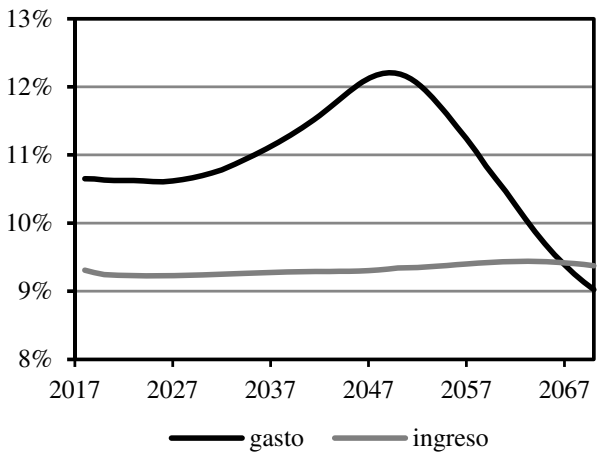

c. Presión fiscal sobre los activos

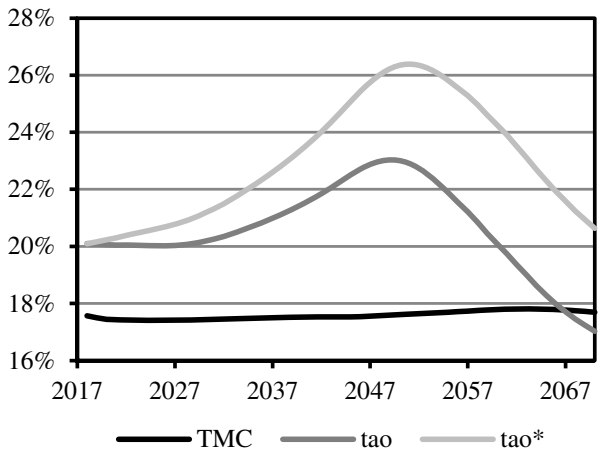

\section{b. Deuda e inmigración acumuladas}

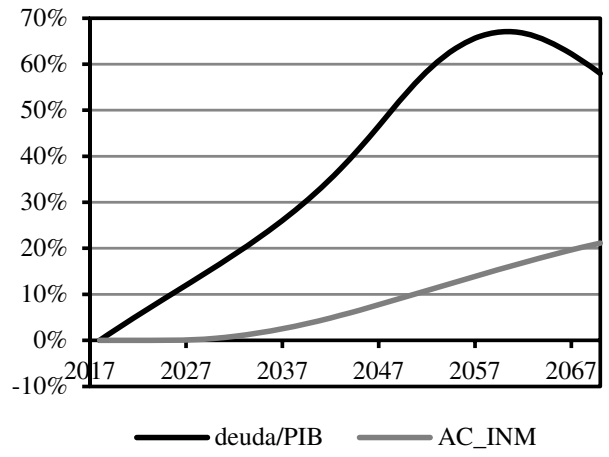

d. Generosidad del sistema

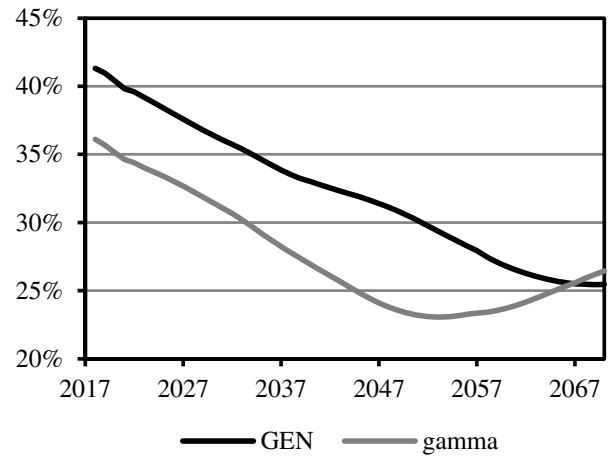

Con la actual política de ingresos, el sistema de pensiones registraría en este escenario un déficit de entre uno y casi tres puntos de PIB hasta 2060. Acumulando estos déficits a un tipo de interés igual a la tasa de inflación más un cuarto de punto, se obtiene la senda de la deuda atribuible al sistema de pensiones que se muestra en el panel $b$ del Gráfico 6, medida también como porcentaje del PIB. La deuda del sistema así calculada es una variable virtual que no tiene necesariamente una contrapartida directa en las cuentas de la Seguridad Social (porque el Estado no tiene por qué canalizar necesariamente sus aportaciones al sistema de pensiones a través de préstamos o aplicar el tipo de interés que estamos suponiendo) pero que resulta útil para visualizar el déficit acumulado de recursos del sistema y la presión que esto supone para el conjunto de las cuentas públicas. Este déficit podría financiarse en parte con impuestos en vez de con deuda del Estado pero ciertamente presionará al alza sobre esta última variable, especialmente cuando el resto de las cuentas públicas ya presentan déficit, como sucede ahora.

En el mismo panel se muestra la inmigración acumulada, medida como fracción de la población residente. Bajo los supuestos del escenario base, la entrada neta de inmigrantes acumulada durante el próximo medio siglo ascenderá a casi un $21 \%$ de la población total del país en 2070. 
Los paneles $c$ y $d$ del Gráfico 6 nos dicen como se repartirían entre los pensionistas y los activos los costes derivados del shock demográfico descrito en la sección anterior. ${ }^{7}$ El panel $d$ muestra la evolución de la generosidad del sistema medida por un indicador, GEN, que se calcula como el ratio entre la pensión media observada en cada momento y la que se habría obtenido aplicando un procedimiento constante de cálculo a una base homogeneizada basada en una media móvil de 30 años del salario real. ${ }^{8}$ En el presente escenario, GEN se reduce gradualmente durante todo el período como consecuencia de los mecanismos automáticos de ajuste establecidos en la reforma del 2013 y de la aplicación gradual de los cambios paramétricos introducidos en la reforma de 2011.

Pese al fuerte descenso de la generosidad del sistema, que alcanza los 11 puntos en 2050, el incremento en el número de pensionistas haría necesario aumentar muy significativamente la presión fiscal sobre los activos para equilibrar las cuentas de la Seguridad Social. La variable $\tau$, cuya senda prevista se muestra en el panel $c$, nos indica precisamente el tipo medio de cotización sobre las rentas totales del trabajo ${ }^{9}$ que sería necesario para igualar los ingresos del sistema al gasto total en pensiones observado en cada ejercicio. En el momento actual, habría que elevar este tipo en unos dos puntos y medio (desde su valor observado, $T M C$, hasta $\tau)$ y seguir subiéndolo gradualmente otros tres puntos de aquí a 2050. Si esto no se hace de forma inmediata, el sistema comenzará a acumular deuda y a sus costes habría que sumarles los correspondientes intereses. La variable $\tau^{*}$ que también se muestra en el panel $c$ indica el tipo medio de cotización que sería necesario para poder pagar con ingresos corrientes las prestaciones del año en curso más los intereses de la deuda acumulada hasta ese punto, así como para devolver el $1 \%$ del principal de dicha deuda. El valor de $\tau^{*}$ en cada año $t$ se calcula suponiendo que las cotizaciones no se tocan entre el momento actual (2018) y $t-1$, esto es, que los tipos medios de cotización siguen la senda prevista y el déficit del sistema se financia con deuda en las condiciones ya indicadas.

Por último, la variable $\gamma$ cuya trayectoria se muestra en el panel $d$ mide el grado de generosidad del sistema que podría financiarse con los ingresos previstos por cotizaciones en el mismo año (TMC). Si se opta por no aumentar las cotizaciones (o los impuestos) y financiar el déficit del sistema con deuda, y si en algún momento la carga de esa deuda se vuelve insostenible y el Reino de España entra en suspensión de pagos, entonces sería necesario llevar a cabo de inmediato un ajuste duro de las pensiones para adaptarlas a los ingresos disponibles. En términos del panel $d$ del Gráfico 6, este escenario correspondería a un salto desde la línea oscura $G E N$ hasta la línea más clara $\gamma$.

Es importante resaltar que, incluso bajo la reforma de 2013, un escenario de ajuste duro de este tipo no sería en absoluto descartable si no se toman pronto medidas para aumentar los ingresos públicos o para contener el gasto. Como ya hemos visto, con los tipos actuales de cotización el sistema de pensiones registraría déficits anuales muy considerables durante varias décadas. Esto se traduciría en una deuda que alcanzaría en 2060 un pico del $67 \%$ del PIB. $\mathrm{Si}$ el Estado no puede financiar estos déficits con ingresos corrientes y una parte significativa de los mismos se añade a la deuda pública ya existente (que en 2018 ronda el $100 \%$ del PIB) junto con la que pudieran generar las demás administraciones públicas de aquí en adelante, una suspensión de pagos no sería descartable una vez los tipos de interés se normalicen. Si 
ésta se produjese en 2050, las pensiones deberían reducirse de golpe en un $23 \%$ para adecuarlas a los ingresos corrientes del sistema -y eso suponiendo que el PIB y las rentas del trabajo no se viesen afectados por la consiguiente crisis, lo que parece altamente improbable-.

\section{b. Supresión de la reforma de 2013 manteniendo el escenario demográfico base}

Manteniendo constantes el resto de los supuestos de nuestro escenario base, y en particular los que tienen que ver con el influjo de inmigrantes, supongamos ahora que se deroga la reforma de 2013, de forma que de 2018 en adelante se vuelve a la actualización de las pensiones con el IPC y no se introduce el factor de sostenibilidad. Comparando el Gráfico 7 con el Gráfico 6 vemos como esta contrarreforma afectaría a las sendas esperadas de las variables de interés. El principal cambio es que la nueva política se traduciría en un sistema mucho más generoso en términos de la cuantía de las pensiones ${ }^{10} \mathrm{y}$, por ello, muy dudosamente sostenible sin forzar cargas muy gravosas sobre ciertas cohortes de futuros activos (y posiblemente otros contribuyentes).

\section{Gráfico 7}

ESCENARIO DEMOGRÁFICO BASE + SUPRESIÓN DE LA REFORMA DE 2013

SUBSISTEMA DE PENSIONES DE LA SEGURIDAD SOCIAL CONTRIBUTIVA

a. Gastos e ingresos/PIB

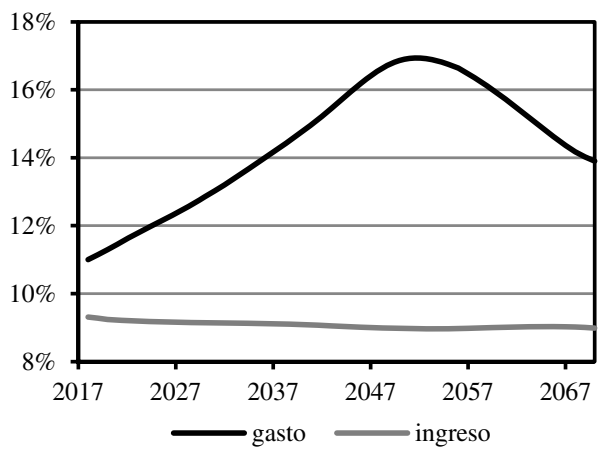

c. Presión fiscal sobre los activos

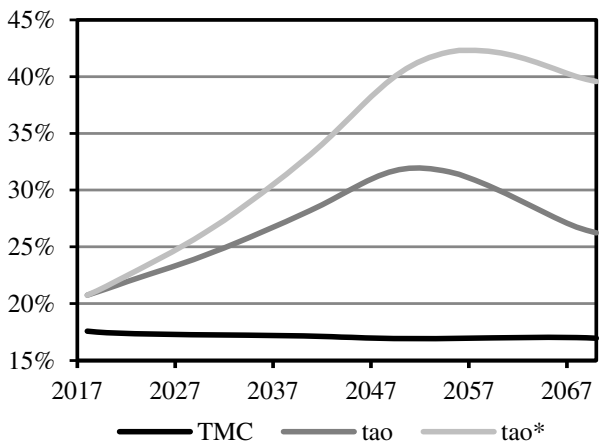

b. Deuda e inmigración acumuladas

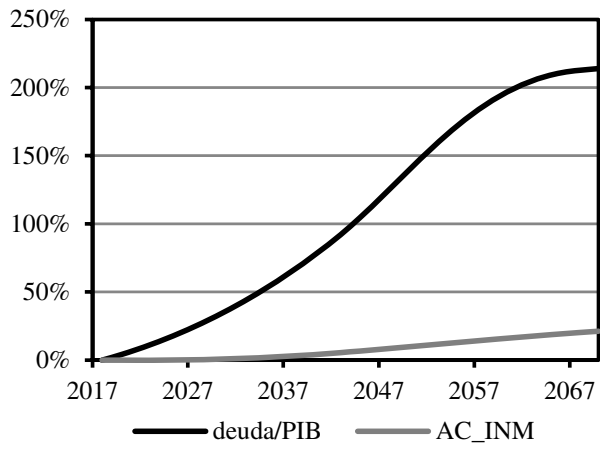

d. Generosidad del sistema

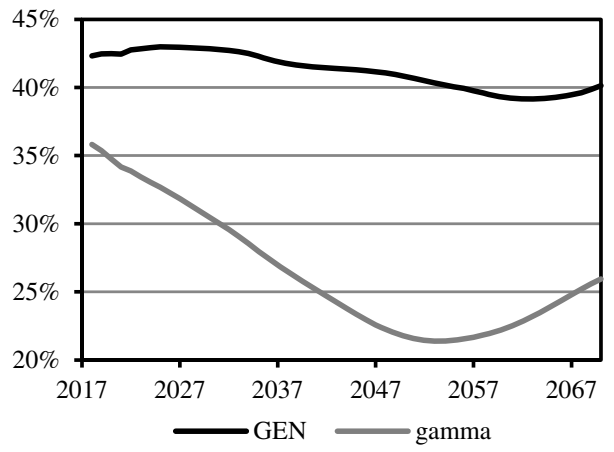

De acuerdo con nuestros cálculos, el gasto en pensiones se dispararía en casi 6 puntos desde el momento actual hasta un pico del 16,9\% del PIB en 2052, lo que, en ausencia de 
una subida de cotizaciones, generaría a finales de la década de los setenta una deuda ligada al sistema de pensiones de más del $200 \%$ del PIB. Para evitar esta situación, muy difícilmente sostenible, habría que elevar sensiblemente las cotizaciones sociales (u otros impuestos). Si el ajuste comenzara inmediatamente, evitando así la acumulación de deuda, hacia el año 2050 sería necesario quedarse con un tercio de las rentas totales del trabajo sólo para pagar las pensiones. Si el ajuste se pospone y se va acumulando deuda hasta 2050, los tipos de cotización necesarios para evitar el colapso se irían por encima del $40 \%$. Y si el colapso llegara y hubiese que ir a un ajuste brusco para adaptarse a los ingresos reales, digamos en 2050, las pensiones tendrían que recortarse de un día para otro en un casi un $50 \%$.

\section{c. Resultados en un escenario de alta inmigración}

En este apartado se rehacen los cálculos anteriores bajo el escenario de inmigración elevada $(A I)$ discutido en la sección 2. Tomando como referencia el año 2050, este escenario supone una entrada acumulada de casi 10 millones de inmigrantes netos desde el momento actual, frente a los 4,3 de nuestro escenario base y los 4,1 del último escenario central de EUROSTAT. Por otra parte, AI estaría bastante próximo a las recientes proyecciones de la AIREF, que van desde 8,9 a 10,4 millones de entradas netas durante el mismo período.

El principal efecto de la fuerte entrada de población en edad de trabajar que caracteriza a este escenario sería un mayor crecimiento del PIB y de los ingresos del sistema de pensiones que mejoraría sensiblemente su situación financiera durante las próximas décadas en relación al escenario base de inmigración moderada. ${ }^{11}$ La mayor entrada de inmigrantes añadiría 7 décimas anuales a la tasa media de crecimiento del PIB entre 2018 y 2050 (que pasaría del $1,2 \%$ al $1,9 \%$ ) y se traduciría en 2050 en un PIB superior en un $25 \%$ al esperado en el escenario base. Aún así, en ausencia de otras medidas, el sistema seguiría en déficit hasta más allá de 2050 aunque se mantuviese la reforma de 2013 y su situación financiera empeoraría de forma muy significativa si ésta se suprimiera. En este último caso, el sistema seguiría siendo difícilmente viable sin un incremento muy significativo de la presión fiscal sobre los trabajadores futuros a pesar del alivio que supondría la elevada inmigración. ${ }^{12}$

\section{Gráfico 8}

ESCENARIO DE ALTA INMIGRACIÓN (AI)+MANTENIMIENTO DE LA REFORMA DE 2013 SUBSISTEMA DE PENSIONES DE LA SEGURIDAD SOCIAL CONTRIBUTIVA

a. Gastos $e$ ingresos/PIB

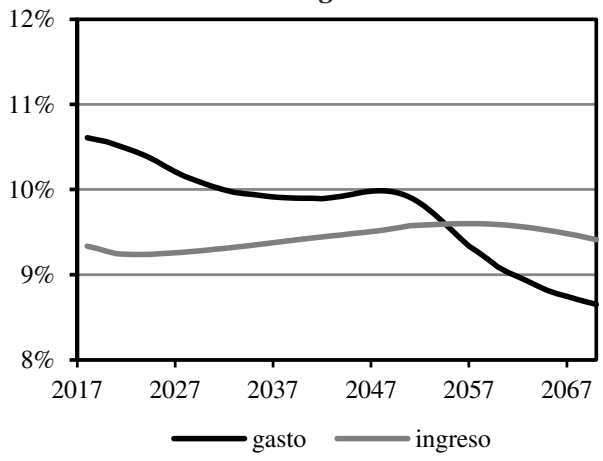

b. Deuda e inmigración acumuladas

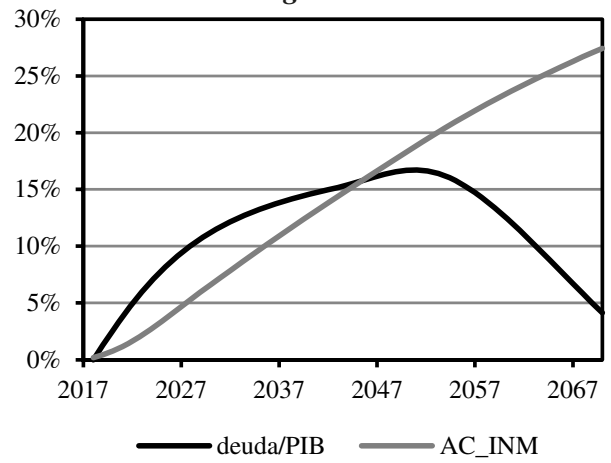


(Continuación.)

c. Presión fiscal sobre los activos

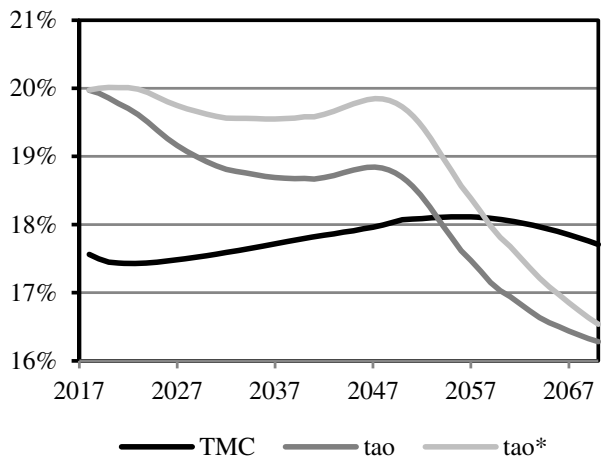

d. Generosidad del sistema

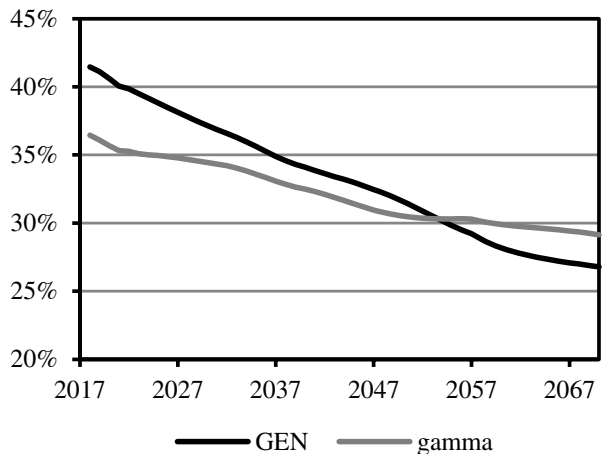

Supongamos en primer lugar que se mantiene la reforma del 2013 y por lo tanto la tónica de contención del gasto en pensiones que fuerzan los mecanismos automáticos descritos más arriba. Las sendas esperadas de las variables de interés bajo este escenario se muestran en el Gráfico 8. El cambio más llamativo en relación al escenario base (Gráfico 6) es que el gasto esperado en pensiones tendería a caer como porcentaje del PIB desde el comienzo del período, y no sólo a partir de 2050 como sucedía en el escenario base. En la misma línea, la presión fiscal sobre los activos que sería necesaria para alcanzar el equilibrio del sistema (tao en el panel $d$ ) también tendería a la baja desde el primer momento. Por otra parte, el sistema seguiría registrando déficits durante casi cuatro décadas y llegaría a acumular una deuda del $17 \%$ del PIB -muy inferior a la esperada en el escenario base- que prácticamente se eliminaría en la parte final del período.

\section{Gráfico 9}

\section{ESCENARIO DE ALTA INMIGRACIÓN (AI)+SUPRESIÓN DE LA REFORMA DE 2013} SUBSISTEMA DE PENSIONES DE LA SEGURIDAD SOCIAL CONTRIBUTIVA

\section{a. Gastos e ingresos/PIB}

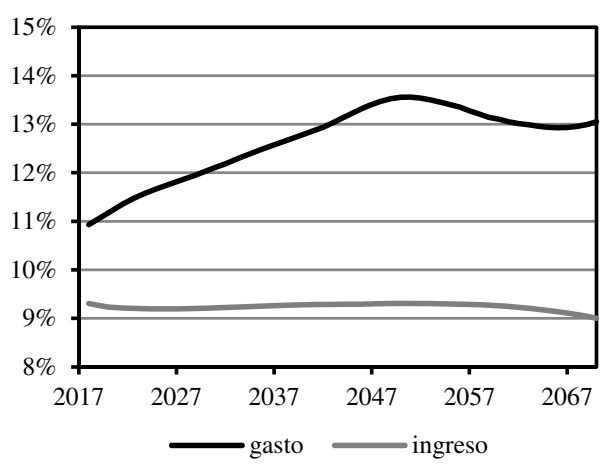

\section{b. Deuda e inmigración acumuladas}

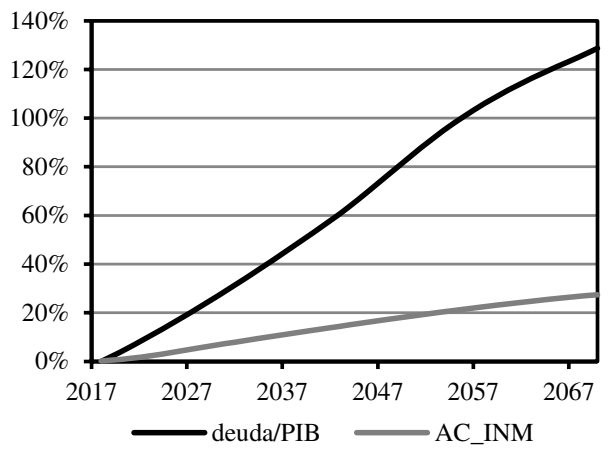




\section{c. Presión fiscal sobre los activos}

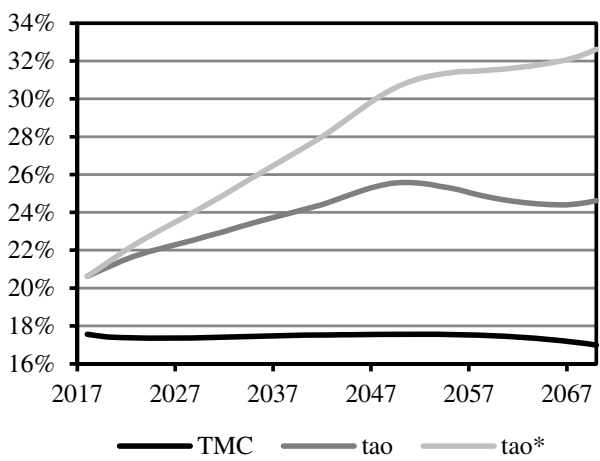

d. Generosidad del sistema

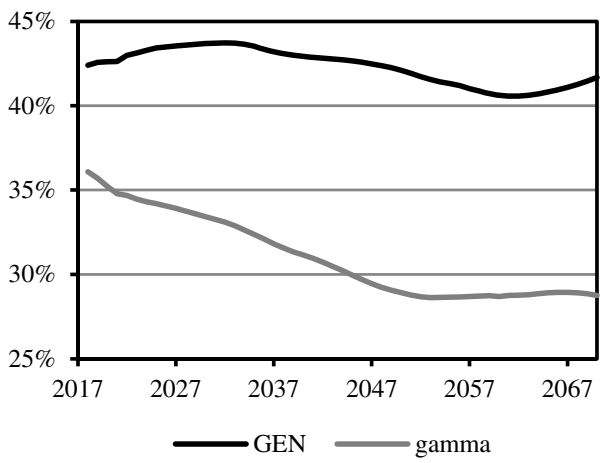

Si partiendo de este escenario suprimimos la reforma del 2013, los resultados esperados son los que se muestran en el Gráfico 9. La senda del gasto en pensiones como fracción del PIB vuelve a ser creciente hasta más allá de 2050 a pesar del mayor crecimiento del denominador y lo mismo sucede con los indicadores de presión fiscal sobre los activos y de deuda acumulada por el sistema de pensiones en ausencia de medidas correctoras, que se dispara por encima del $120 \%$ del PIB. Así pues, aunque los efectos de la supresión de la reforma serían menos dramáticos en el escenario de elevada inmigración, esta política seguiría planteando serios problemas financieros para el conjunto del sector público.

\section{d. Efectos diferenciales de la supresión de la reforma}

A modo de resumen, el Gráfico 10 resume los efectos diferenciales de la derogación de la reforma de 2013 bajo los dos escenarios migratorios que estamos contemplando. El panel $a$ muestra el incremento del déficit del subsistema de pensiones contributivas de la Seguridad Social que resultaría de la derogación de la reforma bajo cada escenario inmigratorio, medido en puntos porcentuales del PIB previsto en el caso de mantenerse la reforma. Como siempre, el déficit del sistema se calcula comparando la previsión de gasto en cada escenario con una proyección de ingresos basada en el supuesto de que se mantienen los tipos actuales de cotización y que el coste de los complementos de mínimos se cubre con transferencias del Estado. El panel $b$ muestra los efectos sobre la deuda acumulada del sistema, medida también como porcentaje del PIB con reforma. Finalmente, el panel $c$ muestra el efecto diferencial de la contrarreforma sobre el indicador $\tau$ que mide el tipo medio de cotización sobre las rentas totales del trabajo que sería necesario para financiar el gasto en pensiones previsto en cada momento. 


\section{Gráfico 10 \\ EFECTOS DIFERENCIALES DE LA SUPRESIÓN DE LA REFORMA DE 2013 SOBRE EL SUBSISTEMA DE PENSIONES DE LA SEGURIDAD SOCIAL CONTRIBUTIVA}

a. Sobre el déficit del sistema/PIB

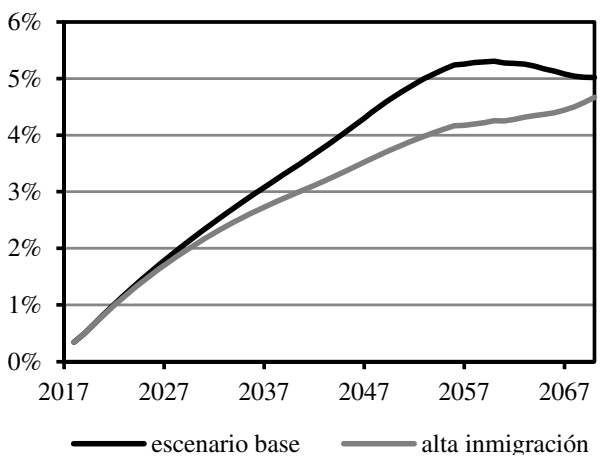

b. Sobre la deuda del sistema/PIB

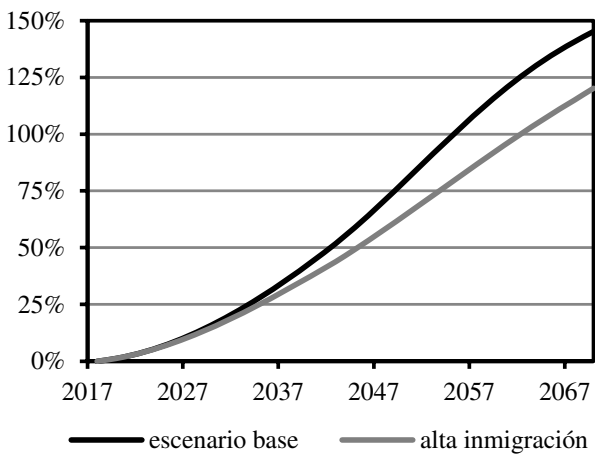

c. Sobre la presión fiscal sobre los activos

$$
\left(\tau_{\text {sin }}-\tau_{\text {con }}\right) / \tau_{\text {con }}
$$

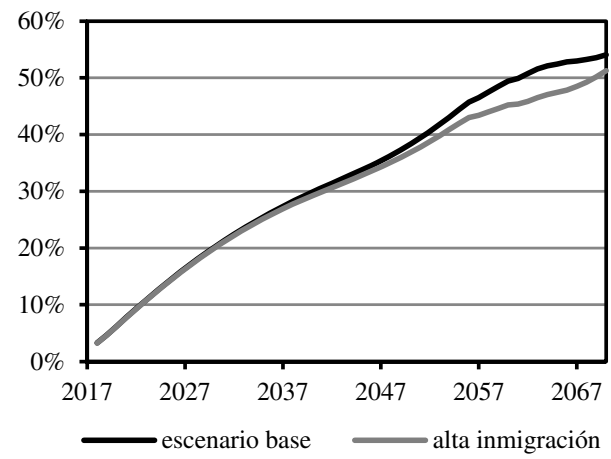

Como se observa en el gráfico, la supresión de la reforma de 2013 incrementa de forma muy notable la presión del sistema de pensiones sobre las finanzas públicas, incluso en el escenario de mayor influjo inmigratorio. Si no se aumentan significativamente las cotizaciones, hacia 2050 la supresión de la reforma añadirá al déficit del sistema entre 4 y 5 puntos de PIB e incrementará su stock de deuda entre un $125 \%$ y un $150 \%$ del PIB. Para evitar esta situación, el incremento de la presión fiscal sobre los activos tendría que ser muy considerable. Si se opta por hacer el ajuste necesario vía cotizaciones, en la parte final del período los tipos tendrán que ser un 50\% más altos si se suprime la reforma de 2013 que si se mantiene.

\section{e. Situación proyectada del sistema en $\mathbf{2 0 5 0}$}

En este apartado se pone el foco en la situación prevista en nuestras proyecciones del sistema de pensiones en el momento más complicado de la transición demográfica, que po- 
demos situar en torno a 2050 con el pico de la tasa de dependencia. El Cuadro 1 muestra los valores proyectados en esa fecha de una serie de indicadores en cada uno de los cuatro escenarios que hemos analizado (inmigración moderada o alta, con o sin la reforma de 2013). Como nota, se añaden también al cuadro los valores proyectados de algunas variables de interés correspondientes al promedio de los años 2020-50.

\begin{tabular}{l} 
Cuadro 1 \\
SITUACIÓN PROYECTADA DEL SISTEMA EN 2050 BAJO DIFERENTES ESCENARIOS \\
\hline \begin{tabular}{rccccc} 
Inmigración $=$ & Base & Base & Alta & Alta \\
Reforma $2013=$ & Sí & No & Sí & No \\
\hline
\end{tabular}
\end{tabular}

1. Recursos necesarios para equilibrar el sistema:
a. en \% del PIB
$2,9-4,7 \quad 8,0-12,6 \quad 0,3-0,9$
$4,3-7,1$
b. en millardos de euros (trasladados a 2017)
$33,7-54,6 \quad 93,0-146,4 \quad 3,9-10,3$
$50,1-82,2$

2. Subida de impuestos necesaria, en $\%$
a. todo en cotizaciones sociales
$31,8-51,4 \quad 87,7-138,0$
$3,7-9,8$
$47,2-77,5$
b. todos los grandes impuestos en misma prop. 11,6-18,8
$32.0-50,5$
$1,3-3,6$
$17,2-28,3$

3. Deuda del sistema scpi, en $\%$ del PIB

54,0

138,4

16,7

83,0

4. Recorte pensiones emerg \% 1-( $\gamma / \mathrm{GEN})$

23,4

46,7

3,3

31,3

5. Pensiones/(RTT-pensiones), $2017=100$

120,3

187,3

92,5

138,1

Nota: Situación proyectada, promedio 2020-50

Recursos necesarios para equilibrar el sistema:
a. en $\%$ del PIB
$1,9-2,8 \quad 4,9-6,8$
$0,7-1,1$
$3,2-4,6$
b. en millardos de euros (trasladados a 2017)
$22,5-32,2 \quad 56,8-79,5$
$8,1-12,7$
$37,5-53,1$

La primera variable de interés son los recursos que serían necesarios para equilibrar las cuentas del sistema de pensiones de la Seguridad Social, calculados a partir de los valores de TMC y $\tau$ o $\tau^{*}$. Esta variable se muestra en primer lugar medida en porcentaje del PIB (de 2050) y se "traslada" después al presente, medida en millardos de euros actuales, multiplicando la variable anterior por el PIB de 2017. De esta forma, podemos hacernos una idea del esfuerzo que sería necesario para cuadrar las cuentas del sistema si estuviéramos ahora en la situación prevista para 2050. Seguidamente, se calcula la proporción en la que habría que subir los tributos actuales para obtener los recursos adicionales calculados en la línea 1.b. El cálculo se realiza en primer lugar suponiendo que sólo se suben las cotizaciones a la Seguridad Social y se repite a continuación suponiendo que los recursos necesarios se obtienen subiendo en la misma proporción las cotizaciones sociales y todos los grandes impuestos (IRPF, Sociedades, IVA e Impuestos Especiales). ${ }^{13}$ Para cada una de estas variables, en el cuadro se ofrece un intervalo que depende, básicamente, de lo que se tarde en iniciar los ajustes necesarios. El límite superior del intervalo se obtiene bajo el supuesto de que el déficit del sistema hasta 2050 se financia enteramente con deuda, mientras que el límite inferior co- 
rrespondería a una situación en la que el sistema llega a 2050 sin deuda gracias a inyecciones de recursos estatales financiadas con incrementos de impuestos.

En la línea 3 se muestra la deuda que el sistema de pensiones acumularía hasta 2050 en ausencia de cambios en la política de ingresos (scpi) de la Seguridad Social y en la línea 4 la proporción en la que habría que recortar las pensiones si en esa fecha se produjese una suspensión de pagos que obligase a adaptar de inmediato las prestaciones del sistema a sus ingresos corrientes. Finalmente, en la línea 5 se muestra el reparto de la renta entre activos y jubilados bajo la hipótesis de que las pensiones se financian sólo con cotizaciones (y la aportación del Estado para los complementos de mínimos). El indicador que se utiliza es el ratio entre el gasto total en pensiones y lo que queda de las rentas totales del trabajo (RTT) tras deducir el coste de las pensiones, normalizado por el valor correspondiente a 2017 (que fue del $24,4 \%$ ).

El análisis del cuadro nos dice que el sistema de pensiones solamente estaría en una posición cómoda en 2050 bajo el escenario que combina una elevada inmigración con el mantenimiento de la reforma de 2013. Bajo estos supuestos, el sistema estaría casi en equilibrio presupuestario y prácticamente libre de deuda, con lo que bastaría una modesta subida de impuestos o de cotizaciones para igualar sus ingresos a sus gastos. En los otros tres escenarios, sin embargo, la situación es mucho más complicada. Aún manteniendo la reforma, si la inmigración está en línea con nuestras proyecciones centrales, en ausencia de medidas de ingreso el sistema presentaría en 2050 un déficit anual de entre 2,9 y 4,7 puntos del PIB y habría acumulado un stock de deuda que podría exceder el $50 \%$ del PIB. Para volver al equilibrio habría que aumentar las cotizaciones al menos en un tercio o las cotizaciones y todos los grandes impuestos al menos en un $12 \%$, o reducir las pensiones de golpe en un $23 \%$. Aún con alta inmigración, la derogación de la reforma nos dejaría en una situación más complicada, con un desequilibrio presupuestario anual de entre 4,3 y 7,1 puntos del PIB que exigiría subidas de cotizaciones de casi el $50 \%$ o recortes de pensiones de más del $30 \%$. Finalmente, la combinación de la supresión de la reforma con una inmigración moderada generaría cifras dramáticas, con un déficit anual de más de 8 puntos de PIB que exigiría subidas de cotizaciones de casi el $90 \%$ o un recorte de las pensiones de casi el $50 \%$. Si nos centramos en la situación promedio entre 2020 y 2050 (resumida en la parte inferior del cuadro), las cifras son menores pero el mensaje es el mismo: en todos los escenarios exceptuando el tercero (con reforma e inmigración elevada), el sistema presentaría un déficit considerable durante varias décadas que exigiría subidas importantes de impuestos o cotizaciones o un rápido crecimiento de la deuda pública que podría llevarnos a una suspensión de pagos.

La fila 5 del Cuadro, por último, ilustra las implicaciones de los distintos escenarios para el reparto de rentas entre activos y pensionistas. Salvo que la inmigración sea muy elevada, el peso de las pensiones frente a las rentas del trabajo netas de cotizaciones tenderá a subir debido al envejecimiento de la población, pero esta subida será mucho mayor si se suprime la reforma. En este escenario, la financiación de las pensiones llegaría a absorber entre un cuarto y un tercio de las rentas totales del trabajo, incluyendo a los autónomos. Las cifras que se muestran en el Cuadro suponen que las pensiones se pagan enteramente con cotizaciones, pero si se desea mantener las mejoras netas de las rentas de los jubilados que estamos consi- 
derando, los recursos tienen que provenir necesariamente de las rentas de los activos, aunque el impacto redistributivo dentro de cada grupo será diferente en función de la combinación de subidas de impuestos y cotizaciones que se utilice. ${ }^{14}$

\section{Conclusión}

En este trabajo se exploran las implicaciones de la supresión de la reforma de 2013 del sistema contributivo de pensiones para las cuentas de la Seguridad Social y del conjunto del sector público y para el reparto de la renta entre activos y pensionistas. Los cálculos se realizan bajo dos escenarios alternativos para analizar la sensibilidad de los resultados a un factor crucial pero muy difícil de predecir con confianza: el influjo neto de inmigrantes durante las próximas décadas.

Nuestros resultados sugieren que un sustancial incremento de la inmigración por encima de las previsiones centrales de Eurostat para España tendría efectos positivos a medio plazo sobre las cuentas de nuestro sistema de pensiones y diluiría los muy considerables costes económicos que comportaría la supresión de la reforma de 2013, pero no hasta el punto de convertir en inocua esta medida. Incluso bajo el escenario migratorio más favorable (e ignorando los posibles efectos adversos de la inmigración sobre la productividad media), la derogación de esta reforma es una decisión que no puede tomarse a la ligera por motivos de equidad intergeneracional y porque supondría incrementar de forma muy significativa la presión que el sistema de pensiones ejerce sobre unas cuentas públicas que todavía registran un déficit importante y un elevado nivel de deuda en el mejor momento del actual ciclo económico y que, además, tendrán que acomodar en el futuro unas necesidades crecientes de gasto en funciones como la sanidad o la dependencia que también son muy sensibles al rápido proceso de envejecimiento de la población en el que nos encontramos.

La contrarreforma que parece estar considerándose en la actualidad comportaría un aumento muy considerable del gasto en pensiones en relación al mantenimiento de la normativa actual, lo que podría terminar de desequilibrar las ya precarias cuentas del sistema durante las próximas décadas hasta hacerlo inviable, abocándolo a un ajuste abrupto en algún momento futuro que comportaría una fuerte reducción de las pensiones de un día para otro y un reparto muy injusto de los necesarios ajustes entre las distintas cohortes de pensionistas. Según nuestros cálculos, en ausencia de otras medidas, este cambio de política añadiría, en promedio durante el próximo medio siglo, entre 3,0 y 3,6 puntos de PIB al déficit anual del sistema de Seguridad Social dependiendo de la evolución de los flujos migratorios, con un pico de más de cinco puntos. Para evitar una espiral de deuda que nos llevaría eventualmente a la suspensión de pagos, resultaría necesaria una inyección de recursos al sistema de este orden de magnitud que podría provenir de una subida de las cotizaciones sociales o de un incremento de las aportaciones del Estado financiadas con mayores impuestos generales. Las subidas de impuestos y/o cotizaciones necesarias para financiar el incremento previsto del gasto tendrían que ser muy significativas, especialmente si se pospone el comienzo del ajuste y se continúa acumulando deuda, y supondrían sin duda una carga muy pesada para determinadas cohortes de trabajadores que sería cuando menos cuestionable en términos de equidad intergeneracional. 
Para evitar esta situación, sería necesario preservar, al menos en parte, los mecanismos de disciplina automática introducidos en la reforma de 2013 o buscar otras alternativas que ayuden a contener el crecimiento del gasto en pensiones, en vez de fiar por entero la viabilidad del sistema a un fuerte incremento de sus ingresos vía impuestos o cotizaciones que, entre otros serios problemas, podría dejarnos sin demasiado margen de maniobra fiscal de cara a la financiación de otras necesidades importantes. ${ }^{15}$ Las posibilidades son muchas y deberían ser objeto de un amplio debate sobre sus méritos relativos en términos de sostenibilidad, equidad intergeneracional y transparencia. Entre las medidas a considerar deberían incluirse, en nuestra opinión, alguna variante del factor de sostenibilidad previsto en la reforma de 2013 o el establecimiento de un nexo automático entre la edad mínima de jubilación y la esperanza de vida en salud, con las salvaguardas necesarias para las ocupaciones de mayor penosidad, así como la extensión gradual del período de cómputo de la pensión a toda la vida laboral. También sería aconsejable establecer una relación estrecha entre las pensiones a percibir y las aportaciones realizadas por cada trabajador, capitalizadas de acuerdo con el crecimiento de la economía. Una posible forma de hacerlo, manteniendo un sistema de reparto, sería la introducción de un sistema de cuentas nocionales con una tasa de capitalización igual a la tasa de crecimiento de la renta agregada.

En cuanto a la revalorización de las pensiones, las mínimas deberían ligarse al IPC con el fin de garantizar la suficiencia del sistema, pero para el resto de prestaciones debería introducirse un índice de revalorización diferente. Este índice debería ser sensible a la situación financiera del sistema, contribuyendo así a su sostenibilidad y a un reparto equilibrado de los costes y beneficios de posibles shocks entre activos y ya jubilados. Una posibilidad a considerar en esta línea sería utilizar como índice de revalorización de las pensiones el producto entre el IPC y el último valor disponible del indicador de salud financiera del sistema de pensiones, definido como el ratio entre sus ingresos y gastos. Esta fórmula suavizaría considerablemente los efectos del IRP, que, en su versión actual y en ausencia de una inyección muy considerable de ingresos, nos abocaría a la cuasi-congelación de las pensiones ya existentes durante varias décadas, pero seguiría contribuyendo a frenar en alguna medida el crecimiento del gasto cuando el sistema está en déficit y trasladaría de manera automática a los pensionistas una parte del incremento de la renta nacional si conseguimos crecer lo suficiente para que el sistema vuelva al superávit.

Una ventaja importante que esta regla de actualización comparte con el IRP y con un sistema de cuentas nocionales bien diseñado es la de hacer explícito y más visible para los ciudadanos el nexo que inevitablemente existe entre la evolución de las pensiones y el crecimiento de la economía. Una de las conclusiones clave de los trabajos en los que se apoya el presente artículo es que la ralentización del crecimiento de la productividad durante las últimas décadas ha jugado un papel muy importante en la gestación de las actuales dificultades financieras de nuestro sistema de pensiones y que revertir esta tendencia ayudaría mucho a equilibrar sus cuentas. Así pues, la mejora de la productividad ha de ser un objetivo prioritario de nuestra política económica, entre otras cosas porque sería la mejor garantía de futuro posible para el estado de bienestar. 


\section{Anexo 1. Una descomposición del saldo financiero del sistema de pensiones}

Para analizar la dinámica del saldo financiero del sistema de pensiones, resulta útil partir de una descomposición del mismo en una serie de factores que recogen la influencia de distintas variables sobre la evolución de sus ingresos y sus gastos. Siguiendo a De la Fuente et al. (2018b), en este anexo se desarrolla una descomposición de este tipo que permite cuantificar el impacto de la demografía, la evolución del empleo y la productividad, la generosidad del sistema, el tipo medio de cotización de las rentas del trabajo y la duración media de la carrera de cotización.

Sea GPENS el gasto total del sistema de pensiones de la Seguridad Social contributiva, (excluyendo, en el presente caso pero no necesariamente, la parte correspondiente de los gastos administrativos de la Seguridad Social), e INGPENS los ingresos del sistema. El indicador de salud financiera que utilizaremos es el ratio entre los ingresos y gastos del sistema,

$$
I S F=\frac{I N G P E N S}{G P E N S}
$$

Para analizar los determinantes inmediatos de la evolución de este ratio, resulta útil proceder como sigue. Sea $W$ el salario medio por ocupado, $L$ el empleo total medido por el número de personas ocupadas y PET la población en edad de trabajar (definida aquí como aquella cuya edad está comprendida entre los 20 y los 64 años). Los ingresos del sistema de pensiones pueden entonces expresarse como

$$
I N G P E N S=\frac{I N G P E N S}{W * L} * W * \frac{L}{P E T} * P E T=T M C * T O C * W * P E T
$$

donde

$$
T O C=\frac{L}{P E T}
$$

es la tasa de ocupación calculada sobre la población en edad de trabajar y

$$
T M C=\frac{I N G P E N S}{W * L}
$$

sería el tipo medio de cotización sobre las rentas del trabajo de los ocupados si éste fuese el único ingreso del sistema -y en la práctica recoge también los ingresos complementarios, como las cotizaciones de los desempleados y las aportaciones del Estado para financiar los complementos de mínimos de las pensiones contributivas-.

Por otra parte, sea NPENS el número total de pensiones vigentes y NRET el número total de personas en edad de jubilación (que, por conveniencia, identificaremos en lo que sigue con los 65 años, aunque la edad legal de jubilación ha variado con el tiempo y se prevé que siga haciéndolo). Entonces, el gasto en pensiones se puede escribir en la forma

$$
\text { GPENS }=\frac{G P E N S}{N P E N S} * \frac{N P E N S}{N R E T} * N R E T=P E N S M * C O B * N R E T
$$


donde PENSM es la pensión media y $C O B$ la tasa de cobertura del sistema de pensiones, esto es, el número de pensiones vigentes por persona en edad de jubilación.

Seguidamente, definamos $\bar{W}$ como el salario medio real por ocupado calculado sobre los 30 años anteriores al ejercicio en curso, utilizando salarios deflactados con el IPC. Esta variable intenta aproximar lo que podríamos llamar la base reguladora media de las pensiones actualmente vigentes, calculada en términos estandarizados, esto es calculada siempre de la misma forma y no sujeta a los cambios que se han ido introduciendo a lo largo del tiempo en las normas de cálculo de la pensión. ${ }^{16}$ Llamando ac a los años medios de cotización del stock de jubilados, el cociente $a c / 40$ sería la parte de la base reguladora que le correspondería como pensión al pensionista medio si se exigiesen 40 años de cotización para una pensión completa y todos los años trabajados se ponderasen de igual forma a efectos del cálculo de la pensión inicial. Esta es simplemente una referencia útil que nos permite definir un factor de generosidad del sistema de pensiones

$$
G E N=\frac{P E N S M}{\frac{a c}{40} \bar{W}}
$$

como el ratio entre la pensión media observada y la que se obtendría aplicando la hipotética norma de cálculo proporcional descrita más arriba a la base estandarizada aproximada que hemos definido antes. ${ }^{17}$

Utilizando (2), (3) y (4), el indicador de salud financiera ISF puede escribirse como

$$
\begin{gathered}
I S F=\frac{I N G P E N S}{G P E N S}=\frac{T M C * T O C * W * P E T}{P E N S M * C O B * N R E T}= \\
\frac{T M C * T O C}{\frac{P E N S M}{\frac{a c}{40} \bar{W}} * \frac{a c}{40} * \frac{W}{W} * C O B * \frac{N R E T}{P E T}}=\frac{T M C * T O C * W E V}{G E N * D U R * C O B * D E P}
\end{gathered}
$$

donde

$$
D E P=\frac{N R E T}{P E T}
$$

es la tasa de dependencia de mayores, esto es, el número de personas en edad de jubilación (65+) que existen por cada persona en edad de trabajar (20-64) y $D U R=a c / 40$ recoge el efecto de la duración de la carrera de cotización media.

El ratio entre el salario medio actual y la media móvil de los salarios medios de los últimos 30 años, ${ }^{18}$

$$
W E V=\frac{W}{\bar{W}}
$$

es lo que denominaremos el componente de evolución del salario real del ISF. Este factor juega un papel crucial en la salud financiera del sistema porque los ingresos de éste dependen del salario actual, $W$, mientras que sus gastos son una función de los salarios existentes 
durante muchos años pasados. Obsérvese que WEV depende directamente de la tasa de crecimiento de los salarios reales y por lo tanto, en última instancia, de la tasa de crecimiento de la productividad. Así, si los salarios reales se mantienen constantes, WEV será igual a la unidad, mientras que si éstos aumentan con el paso del tiempo, el promedio de los últimos 30 años será inferior al sueldo medio actual, lo que implica valores de $W E V$ mayores que uno y crecientes en la tasa de crecimiento del salario medio real. Así pues, cuanto más rápido crezca la productividad, y con ella los salarios reales, mejor será la salud financiera del sistema pues el pago de las pensiones requerirá una fracción menor de las rentas salariales actuales, que son la base que soporta las cotizaciones sociales.

A modo de resumen, el $I S F$ se puede descomponer en una serie de factores, tal como se indica en la siguiente expresión:

$$
I S F=\frac{I N G P E N S}{G P E N S}=\frac{T M C * T O C * W E V}{G E N * D U R * C O B * D E P}
$$

Para cada uno de los escenarios que simulamos, el modelo permite calcular los distintos términos que aparecen en esta expresión, ofreciéndonos así una radiografía de los factores que inciden sobre la evolución de las cuentas del sistema en cada caso.

A partir de esta expresión podemos definir y calcular también dos términos de interés que se utilizan en el texto. El primero, al que denominaremos $\tau$, es el tipo medio de cotización sobre las rentas totales del trabajo que igualaría los ingresos del sistema contributivo de pensiones a sus gastos, haciendo por tanto que el $I S F$ sea igual a la unidad. Por definición, $\tau$ satisface la siguiente expresión,

$$
\frac{\tau * T O C * W E V}{G E N * D U R * C O B * D E P}=1
$$

de donde

$$
\tau=\frac{G E N * D U R * C O B * D E P}{T O C * W E V}=\frac{G E N * D U R * C O B * D E P}{T O C * W E V * T M C} * T M C=\frac{T M C}{I S F}
$$

lo que nos permite calcular $\tau$ directamente a partir del ISF y del tipo medio de cotización observado. El segundo término de interés, al que llamaremos $\gamma$, es el indicador de generosidad del sistema que podría mantenerse sólo con sus ingresos corrientes, esto es, el valor de GEN que hace que $I S F$ sea igual a la unidad en (6). Procediendo como arriba, tenemos

$$
\frac{T M C * T O C * W E V}{\gamma * D U R * C O B * D E P}=1
$$

de donde

$$
\gamma=\frac{T M C * T O C * W E V}{D U R * C O B * D E P}=\frac{T M C * T O C+W E V}{D U R * C O B * D E P * G E N} * G E N=I S F * G E N
$$




\section{Anexo 2. Supuestos utilizados en el escenario base y datos de referencia para la calibración}

\section{Demografía}

Fecundidad

Curvas de fertilidad por edad y año: proyección Eurostat $2013 \rightarrow$ ICF $1.32(2013)$ a $1.55(2060)$

Mortalidad

Curvas de mortalidad por edad, género y año: proyección Eurostat $2013 \rightarrow$ EVmujeres 85.2 (2013) a 90(2060).

Migraciones (base)

Flujos de inmigrantes netos por edad y año: Eurostat 2013. Flujos agregados netos: -310(2013) 6.4 (2025) 305 (2050) 275 (2060) media:110 .

Migraciones (Alta Inmig) -171(2013) 318 (2025) 305 (2050) 275 (2060) Media: 295.

Educación

Distribución (4 grupos) por género y año: constante durante la proyección en el valor observado individuos 30/35 años en 2011 en ECV 2004/2011.

\section{Mercado de Trabajo}

Ingresos laborales

Perfiles de unidades de trabajo eficiente por edad ( para cada grupo de educación y género) estimados ECV 2004/2011 se mantienen desplazan con años (crecimiento exógeno de la productividad del trabajo).

Tasas laborales agregadas

Actividad y desempleo: 2014/2018 usan proyección Plan Estabilidad 2014/2018. Resto de los años: convergencia a Proyección AWG 2015 (por género). Tasas macro se desagregan por educación, género y cohorte conforme lo observado en EPA 2013.

Tasas actividad $(20 / 64)$

81.9 (2020) a 85.2 (2060); Para mujeres 78.6 (2020) a 84.8 (2060).

Tasas de desempleo

$19.0(2020)$ a $7.3(2060)$.

Tasa de empleo (20/64)

$66.3(2020)$ a $79(2060)$.

Jubilación

Cohortes nacidas antes de 1943: datos MCVL 2011 por género y cohorte. Jubilación endógena para cohortes nacidas 1980 y siguientes. Cohortes intermedias: convergencia progresiva a la distribución endógena.

Edad media de jubilación

64.2 (2018) 66.8 (2040) 67.9(2060) 66.8 (2070).

\section{Sistema de pensiones}

Tasas de cobertura

Construidas a partir de observaciones iniciales en MCVL y crecimiento de las tasas empleo del modelo. Senda: 1.15 (2020) 1.06 (2040) 1.10 (2060).

Cotizaciones máximas/ mínimas

Se mantienen constantes en términos reales en valores observados 2015.

Pensiones máximas/mínimas

Indexadas IRP (simulación base) o a inflación(eliminación reforma 2013).

Pen. jubilación/viudedad Endógenas. 
(Continuación.)

\section{Sistema de pensiones}

Pen. Incap.Permanente,

Orfandad y Favor familiar

Pen. Reg. Clases Pasivas

Fondo de Reserva
Varones: se mantienen las tasas de incidencia por edad de 2015; mujeres: convergencia progresiva a las tasas de varones. Valores medios indexados como pensiones de jubilación y viudedad.

Agotamiento natural del sistema tras cierre a nuevas altas en 2010.

Regla de dotación: superávit sistema se carga $100 \%$ al Fondo; Déficit: se pagan 2 pagas extra. Agotado en 2017, se reactiva 2062 (simulación base).

\section{Resto Sector Público}

Consumo público

Otras trasferencias y gastos

Deuda Pública

Sendas gasto (\% PIB) se toman de la proyección del AWG 2015 (2020 a 2060: Sanidad (6.2 a 6.9), educación (4.1 a 3.7) y dependencia (1.2 a 2.4) .

Desempleo: proyección AWG2015: 1.6 (2020) a 0.5 (2060).

Resto: gasto constante en \%PIB en el valor observado en 2015.

Progresiva reducción \% PIB desde valor observado 2015 a $72 \%$ en 2050.

\section{Macroeconomía}

Inflación

Tasa crecimiento productividad trabajo
Recuperación progresiva desde valor observado en 2015 al $2 \%$ en 2020 .

Ajuste desde escenario del Plan de Estabilidad 2014/2018 a la proyección AWG 2015: 0.2 (2019) 1.1 (2025) 1.3(2030) 1.5 (2035 y siguientes). 


\section{Notas}

1. Véanse las proyecciones de población en https://ec.europa.eu/eurostat/data/database?node_code=proj.

2. La tasa de ocupación de la población total, definida como el ratio entre el empleo total de todas las edades y la población entre 20 y 64 años, aumentaría muy significativamente hasta acercase al $80 \%$, ganando casi 21 puntos durante el mismo período y dejándonos con una tasa de paro en torno al $7 \%$. Estamos suponiendo, por tanto, que los problemas actuales del mercado de trabajo español desaparecen gradualmente durante las próximas décadas y que convergemos a niveles de actividad y ocupación a los que ahora sólo se acercan ciertos países del norte de Europa.

3. Véase especialmente De la Fuente, García y Sánchez (DGS, 2017 y 2018 a y b), así como Sánchez (2017 a y b) para los detalles más técnicos del modelo y su calibración. La principal ventaja de los modelos de equilibrio frente a otras técnicas de modelización del gasto en pensiones es que estos garantizan la coherencia entre las decisiones individuales y los agregados macroeconómicos. Estos modelos permiten analizar la respuesta de los agentes (en sus decisiones de ahorro y jubilación) ante cambios de política y cuantificar las implicaciones de estos para el bienestar.

4. Se supone que de ahora a 2020 la inflación converge al $2 \%$ y el crecimiento del factor de productividad del trabajo al 1,5\% (a lo que habría que sumar las mejoras de la productividad derivadas del aumento previsto en el nivel educativo). De esa fecha en adelante, se supone que las dos variables se mantienen constantes. Como referencia, la tasa media de crecimiento del producto real por ocupado en España entre 1990 y 2015 fue del $0,90 \%$ anual.

5. Para un análisis del caso español centrado en la sensibilidad de los resultados a diversas hipótesis sobre la evolución de las variables exógenas más importantes, véase Doménech y Melguizo (2009). Estos autores sugieren una forma interesante de ilustrar los efectos acumulativos de distintas fuentes de incertidumbre sobre el posible abanico de resultados partiendo de una descomposición contable del gasto en pensiones.

6. Sobre esta cuestión véanse las secciones 3.5 y 4 de SGD (2018a). En lo que sigue nos centraremos en cuestiones de sostenibilidad y equidad intergeneracional que aconsejan mantener al menos en parte la reforma 2013, pero ésta también plantea un problema claro de insuficiencia a través de la progresiva pérdida de poder adquisitivo de las pensiones más bajas que habría que abordar si se mantiene el IRP. Nuestra recomendación en este sentido es la de indexar al IPC las pensiones mínimas, pero no necesariamente el resto.

7. Más generalmente, habría que hablar de contribuyentes en vez de activos, pues parte del coste de las pensiones puede financiarse con impuestos sobre rentas distintas de las del trabajo, incluyendo las propias pensiones, o sobre otras bases impositivas. Hay por tanto más actores de los que aquí se mencionan, pero por el momento centraremos la atención en los más directamente implicados.

8. Para más detalles, véase el Anexo 1.

9. Esta variable intenta aproximar la base sobre la que recaen las cotizaciones sociales. Su valor inicial se toma de De la Fuente (2017) e incluye una estimación de las rentas salariales que corresponderían a trabajadores autónomos y otros no asalariados. Para proyectarla al futuro, se utiliza la proyección del PIB que general el modelo y se supone que el peso de las rentas del trabajo en el PIB se mantiene constante.

10. Mirando el panel $d$ del Gráfico 7, puede resultar chocante que el indicador de generosidad del sistema se mantenga aproximadamente estable, o incluso aumente ligeramente, durante los primeros años del período a pesar de la gradual implementación de los cambios paramétricos introducidos en la reforma de 2011. Esto se debe en parte a los largos períodos transitorios fijados en la reforma (y al hecho de que nuestro modelo no contempla fracciones de año, con lo que por ejemplo la edad normal de jubilación pasaría directamente de 65 a 66 años en 2021 y a 67 en 2027) y en parte a la reacción de los agentes a la reforma, y en particular al retraso en su edad media de jubilación, que tiende a aumentar la generosidad del sistema al evitar parte de las penalizaciones ligadas a una jubilación temprana.

11. En términos de ratios sobre PIB, la mejora tomaría la forma de un menor ratio de gasto como consecuencia del mayor crecimiento del denominador, mientras que el ratio de ingreso se mantendría aproximadamente constante. 
12. Por otra parte, el incremento de la inmigración en este escenario se traduce en un incremento del número de pensionistas futuros. Esto hace que el ratio de gasto en pensiones se reduzca mucho menos en la parte final del período en el escenario de elevada inmigración que en el escenario migratorio base, con o sin la derogación de la reforma.

13. Para realizar estos cálculos hemos utilizado datos de la Seguridad Social y de la Agencia Tributaria correspondientes a 2017. Aunque en el texto se habla de cotizaciones sociales para abreviar, se incluyen aquí también las aportaciones del Estado para complementos de mínimos y los ingresos patrimoniales y por tasas de la Seguridad Social y se sustrae del total el coste de las prestaciones contributivas distintas de las pensiones, llegando así a una cifra de 106.112 millones de euros en "cotizaciones netas". La recaudación de los impuestos citados se toma del informe de recaudación de la AEAT (2018) y asciende a 290.248 millones.

14. Para hacerse una idea de la posible incidencia de las subidas impositivas sobre activos y jubilados, los siguientes datos pueden ser útiles. En 2017 las rentas totales del trabajo ascendían a 625.220 millones (véase la nota al pie número 7). Restando esta cifra del PIB según CNE, se obtiene un excedente bruto de explotación de 429.643 millones, de lo que hay que deducir el consumo de capital fijo (202.816 millones) para obtener una estimación de 226.827 millones para las rentas netas del capital. Por otra parte, los ingresos por pensiones contributivas de la Seguridad Social en el mismo año ascendieron a 122.772 millones. La suma de los tres conceptos asciende a 974.819 millones, de los que el 64,1\% corresponde a las rentas netas del trabajo. De acuerdo con la ECV, finalmente, casi el $40 \%$ de las rentas del capital corresponden a hogares cuyo sustentador principal tiene 65 años o más.

15. Algunos políticos y analistas sostienen que es posible absorber sin excesivos problemas incrementos del gasto en pensiones del orden de los que aquí estimamos. El argumento suele basarse en la observación de que España está significativamente por debajo de la media europea en términos del peso de sus ingresos tributarios en el PIB. Según datos recientes de la Comisión Europea (véase García, 2018, sección 3.3), España estaba en 2016 algo más de siete puntos por debajo de la media de la zona euro en términos de este indicador de presión fiscal, de lo que tres puntos reflejaban unas cotizaciones más bajas que la media por parte de los trabajadores. Dado el déficit actual del sistema de pensiones (1,6 puntos de PIB) y las previsiones sobre su evolución recogidas en el Cuadro 1, las pensiones absorberían el grueso del posible margen tributario que supone el diferencial de presión fiscal con la media europea, lo que dejaría pocos recursos para atender otras cuestiones importantes. Además, no conviene minusvalorar ni la dificultad ni los posibles efectos adversos de un aumento en la presión fiscal de esta magnitud.

16. La pensión inicial se calcula como un porcentaje de la llamada base reguladora de la pensión. Esta base es una media de salarios pasados reflactados con el IPC calculada durante un determinado período y el porcentaje es una función no lineal de los años de cotización. Algunos detalles de estos cálculos han cambiado varias veces en las últimas décadas.

17. El factor de generosidad se ha definido con las pensiones de jubilación en mente. Aunque éstas son la principal partida de gasto, la existencia de otros tipos de pensiones ligadas a las de jubilación pero con cuantías inferiores distorsiona en alguna medida el valor de esta variable y exige una cierta precaución a la hora de interpretarla.

18. El salario medio se calcula dividiendo las rentas totales del trabajo por el número de ocupados. Los datos de ambas variables provienen de De la Fuente (2017), donde se ajusta al alza la remuneración de los asalariados que ofrece la Contabilidad Regional y Nacional para aproximar lo que serían las rentas totales del trabajo, incluyendo las correspondientes a los no asalariados. Con este fin, se imputa a cada trabajador no asalariado el salario medio de los asalariados en el mismo sector y región, trabajando con el máximo detalle sectorial disponible en la Contabilidad Regional de España. La serie resultante se divide el número de ocupados para calcular el salario medio y construir luego la media móvil sobre 30 años de esta variable.

\section{Referencias}

Agencia Tributaria, AT (2018), Informe anual de recaudación tributaria. Año 2017, Madrid, http://www. agenciatributaria.es/AEAT.internet/datosabiertos/catalogo/hacienda/Informes_anuales_de_Recau dacion_Tributaria.shtml. 
Autoridad Independiente de Responsabilidad Fiscal, AIREF (2018), Previsiones demográficas: una vision integrada, http://www.airef.es/-/la-airef-preve-que-la-poblacion-espanola-aumente-entre-4-y13-millones-en-30-anos-y-se-situe-entre-51-y-60-millones-de-habitantes-en-2050.

De la Fuente, A. (2017), "Series largas de algunos agregados económicos y demográficos regionales: Actualización de RegData hasta 2016 (RegData y RegData Dem versión 5.0-2016)”, FEDEA, Estudios sobre la Economía Española, 2017/26, Madrid, http://documentos.fedea.net/pubs/eee/eee201726.pdf.

De la Fuente, A., García, M. A. y Sánchez, A. (DGS) (2017), "La salud financier del sistema publico de pensiones español. Análisis retrospective, proyecciones de largo plazo y factores de riesgo", FEDEA, Estudios sobre Economía Española, 2017-04, Madrid, http://documentos.fedea.net/pubs/eee/eee201704.pdf.

De la Fuente, A., García, M. A. y Sánchez, A. (DGS) (2018 a), "La Salud Financiera del Sistema Público de Pensiones Español: Proyecciones de Largo Plazo y Factores de Riesgo", Hacienda Pública Española/Review of Public Economic, 229(2):123-156.

De la Fuente, A., García, M. A. y Sánchez, A. (DGS) (2018b), “An accounting decomposition of the net financial balance of the public pension system with an application to Spain, 1985-2017", Revista de Economía Aplicada 78: 5-19.

Domenech, R. y Melguizo, A. (2009), "Projecting pension expenditure in Spain: on uncertainty, communication and transparency", en Franco, D. (ed.), Fiscal Sustainability: Analytical Developments and Emerging Policy Issues, Banca d'Italia, Roma, 707-728.

European-Commission, EC (2014), "The 2015 Aging Report. Underlying assumptions and projection methodologies", European Economy 8/2014, http://ec.europa.eu/economy_finance/publications/ european_economy/2014/pdf/ee8_en.pdf.

European-Commission, EC (2015), “The 2015 Aging Report. Economic and budgetary projections for the 28 EU Member States (2013-2060)", European Economy 3/2015, http://ec.europa.eu/economy_ finance/publications/european_economy/2015/pdf/ee3_en.pdf.

García Díaz, M. A. (2019), "El sistema público español de pensiones: Mitos y realidades”, FEDEA, Estudios sobre Economía Española, 2019-01, Madrid, http://documentos.fedea.net/pubs/eee/eee201901.pdf.

Instituto Nacional de Estadística, INE (2018 a), Cifras de población y censos demográficos, en Base de datos electrónica INEbase, Demografía y población, Madrid, http://www.ine.es/inebmenu/mnu_ cifraspob.htm.

Instituto Nacional de Estadística, INE (2018 b), Indicadores demográficos básicos, en Base de datos electrónica INEbase, Demografía y población, Análisisyestudios demográficos, Madrid,http://www.ine.es/ inebmenu/mnu_analisis.htm.

Ministerio de Empleo y Seguridad Social, MEySS (varios años), Presupuestos de la Seguridad Social, Informe Económico-Financiero, http://www.seg-social.es/Internet_1/Estadistica/PresupuestosyEstudi 47977/Presupuestos/PresupSegSocialanniosant/PresupSegSoc2014/Informacioncomplementaria/ index.htm.

Sánchez Martín, A. (2017 a), "Proyecciones financieras y de bienestar del sistema español de pensiones: modelización en equilibrio general”, FEDEA, Estudios sobre la Economía Española, 2017-03, Madrid, http://documentos.fedea.net/pubs/eee/eee2017-03.pdf. 
Sánchez Martín (2017b), "Proyecciones financieras y de bienestar del sistema español de pensiones: resultados de simulación”, FEDEA, Estudios sobre la Economía Española, 2017-15, Madrid, http:// documentos.fedea.net/pubs/eee/eee2017-15.pdf.

\begin{abstract}
This paper analyzes the likely consequences of the reversal of the 2013 pension reform. We construct projections of the revenues and expenditures of the contributive Social Security pension system until 2010 with and without such reform and explore its implications for public finances and for the distribution of income between active workers and pensioners under two alternative immigration scenarios.
\end{abstract}

Keywords: pension reform in Spain, sustainability.

JEL Classification: H55, J11. 
